\title{
THE ROLE OF ATTRIBUTIONS IN ABSTINENCE, LAPSE, AND RELAPSE FOLLOWING SUBSTANCE ABUSE TREATMENT
}

\author{
MAUREEN A. WALTON \\ Alcohol Research Center, University of Michigan
}

FELIPE G. CASTRO

Department of Psychology and Hispanic Research Center. Arizona State University

\section{ELIZABETH H. BARRINGTON}

Department of Physical Education and Health, San Diego Mesa College

\begin{abstract}
This study examined the role of attributions in the lapse and relapse process following substance abuse treatment. According to Marlatt and Gordon's theoretical framework. attributions made after a lapse (e.g., the Abstinence Violation Effect [AVE]) determine whether it progresses to a relapse. Also examined were the attributions of recovering drug users who were tempted but remained abstinent (never lapsed). Ninety-seven participants were recruited from an inpatient treatment center for substance abuse and completed an interview 6 months after leaving treatment. Findings partially confirmed predictions made by the AVE. Predictions made by the AVE were not supported in that lapsers and relapsers were similar regarding their internal/external attributions following a return to drug use; predictions were supported as relapsers made more stable and global attributions as compared to lapsers. Also as predicted, abstainers made more internal, stable. and global attributions regarding their abstinence (as compared to lapsers following their slip). Abstainers attributions for their success in remaining abstinent tended to be similar to the attributions made by relapsers for their failure to remain abstinent (i.e., for their relapse). Combined, these findings highlight the complexity of the attributional process in early recovery from substance abuse. Clinical and research implications of the findings are discussed in relation to substance abuse relapse prevention.
\end{abstract}

Regardless of substance used, relapse rates following substance abuse treatment are alarmingly high (typically $75 \%$ ) in the 3- to 6-month period following treatment (Brownell, Marlatt, Lichtenstein, \& Wilson, 1986; Hubbard \& Marsden, 1986; Hunt, Barnett, \& Branch 1971; Marlatt \& Gordon, 1985; Wallace, 1989; Washton, 1986). Research attempting to explain this high relapse rate has been guided by Marlatt and Gordons' (1985) theory, in which relapse is viewed as a process that is determined by cognitive and situational factors. Marlatt and Gordon have hypothesized that a recovering substance user maintains the expectation of control while abstinent, until he or she encounters a high-risk situation. The probability of an initial use in response to a high risk situation is affected by the person's expectations about the consequences associated with using psychoactive substances. Whether an initial use of a prohibited substance (a lapse) will progress to more intense drug or alcohol use (a relapse) in part depends upon attributions made in reaction to the lapse.

Substance abuse research had drawi upon the major components of attribution theory to examine the attributions of lapsers and relapsers. These major components are: locus of causality (internal/external), stability (stable/unstable), and situational specificity (global/specific) (Abramson, Garber, \& Seligman, 1980; Weiner, 1974).

This research is partially supported by National Institute on Drug Abusc Grant DA05661.

Requests for reprints should be sent to Maureen A. Walton, University of Michigan, Alcohol Research Center, 400 E. Eisenhower Parkway, Building 2. Suite A. Ann Arbor, MI 48108. 
When these attributions occur in a particular maladaptive pattern, they are termed the Abstinent Violation Effect ( $A V E$ ), and are thought to promote relapse (Marlatt \& Gordon, 1985). For example, relapse would most likely result if a recovering drug or alcohol user would make the following attributions upon experiencing a lapse: (a) they perceive the cause of the lapse as originating in themselves (an internal attribution), (b) they perceive that the lapse is trait-like and will occur in similar situations in the future (a stable attribution), and (c) they perceive that the lapse will generalize to other cues (a global attribution). In contrast, under a condition of lapse, persons least likely to relapse would be those who perceive that their lapse is promoted by other people or circumstances (an external attribution), that the lapse is an isolated instance and thus is not likely to occur in similar situations in the future (an unstable attribution), and that the lapse occurs in relation to a particular cue (a sperific attribution).

Marlatt and Gordons' (1985) formulation did not specifically address the attributional patterns of persons who are tempted yet who maintain abstinence. By implication however, those who initially succeed in avoiding a lapse would be likely to continue to succeed if they made internal attributions (by perceiving their abstinence as a personal success), stable attributions (by thinking that their abstinence will persist across time), and global attributions (by believing that their abstinence is generalizable to other substances) for their success. Table 1 summarizes the postulated attributional profiles of abstainers, lapsers, and relapsers. The purpose of this study was: (a) to develop precise and conceptually meaningful operational definitions of lapse and relapse, (b) to validate the theorized role of attributions (and the Abstinence Violation Effect) in determining lapse and relapse, and (c) to examine the attributions of abstainers for their success in maintaining sobriety.

\section{AVE/Atribution literature}

The literature focusing on the attributional patterns of cigarette smokers tends to support the attributional profile explained by the Abstinent Violation Effect. Two studies among smokers distinguished lapse and relapse by defining lapse as a slip followed by a period of abstinence, and relapse as a slip followed by a period of use. Curry, Marlatt, and Gordon (1987) found that relapsers reported making more internal, stable, and global attributions than did lapsers (those who regained abstinence). Similarly, O'Connell and Martin (1987) found that smokers who lapsed made external attributions for their cigarette use, whereas smokers who relapsed made more internal attributions. Both of these results support the tenets of the AVE. When reuse was examined (lapse and relapse were not distinguished), reuse was associated with internal, stable, and global attributions about a return to smoking (Goldstein.

Table 1. Hypothesized relationships between attributions and outcome category after exposure to a tempting situation

\begin{tabular}{|c|c|c|c|}
\hline \multirow{2}{*}{$\begin{array}{l}\text { Attributional } \\
\text { dimension }\end{array}$} & \multicolumn{3}{|c|}{ Outcome } \\
\hline & Abstainers & Lapsers & Relapsers \\
\hline Locus of causality & Internal & External & Internal \\
\hline Stability across time & Stable & Unstable & Stable \\
\hline Substance specificity & Global & Specific & Global \\
\hline
\end{tabular}


Gordon, \& Marlatt, 1984); this finding is consistent with predictions made by the AVE if the participants relapsed, as opposed to lapsed.

Data from studies of the attributions of alcoholics and illicit drug users are less supportive of the AVE than are data from studies of smokers. This literature is convoluted by the use of generic attribution measures and by a failure to clearly distinguish lapse and relapse. Bradley, Gossop, Brewin, Phillips, and Green (1992) measured opiate addicts' attributions for hypothetical negative outcomes, and 6 months later measured the occurrence of lapses and relapses. Lapse was defined as some use but abstinent at time of follow-up interview, and relapse was defined as daily use at follow-up. Contrary to expectations, these investigators found that lapsers and relapsers did not differ in respect to their stable and global attributions they made 6 month's earlier for hypothetical negative outcomes. Furthermore, lapsers made more internal attributions than did relapsers, which is inconsistent with the predictions of the AVE.

Two studies did not distinguish between lapsers and relapsers. Birke, Edelmann, and Davis (1990) did not find differences between the causal, stable, or specific attributions of abstainers and reusers in a sample of illicit drug users. These authors concluded that the AVE may not apply to illicit drug users, due to their different lifestyles and to the consequences that accompany illegal drug use versus cigarette use. In contrast, Gutierres and Reich (1988) found that a change from treatment to follow-up from external to internal attributions predicted male illegal drug users' rehabilitation at follow-up (when rehabilitation was measured as social functioning and reuse); however, this relationship was not found for female illegal drug users. The authors did not distinguish whether the rehabilitation group consisted of abstainers and/or lapsers. Their findings may support the predictions made by the AVE regarding internal attributions if the "rehabilitation" group consisted of abstainers and not lapsers.

Other research suggests that shifts in attributions may explain differences in the alcoholics' attributions. McKay, O'Farrell, Maisto, Connors, and Funder (1989) considered the temporal relationship between the measurement of attributions and the first episode of reuse among male alcoholics. Participants whose first reuse occurred within the previous 8 months (recent users) made more situational attributions (external), while participants whose first reuse occurred prior to the previous 8 months (past users) made more dispositional attributions (internal). The authors suggested that making situational attributions is a way to alleviate the shame that accompanies relapsing; they hypothesized that over time, the memory becomes less painful so it is easier for the alcoholic to make dispositional attributions. An alternative explanation is that the recent users group consisted primarily of lapsers, while the past users group consisted primarily of relapsers. Evidence in support of this explanation is that recent use episodes ranged from 1 to 60 and past use episodes ranged from 2 to 730 .

Alternatively, the lack of clarity regarding the role of attributions and the AVE in differentiating lapse and relapse among substance users may be explained by which attributional messages are included in the treatment program. For example, in studies of cigarette smokers and alcoholics, participants' attributions were successfully manipulated by randomly assigning participants to programs focusing on internal responsibility or external responsibility (Harackiewicz, Sansome, Blair, Epstein, \& Manderlink, 1987; Maisto, O’Farrell, Connors, McKay, \& Pelcovits, 1988). Participants assigned to traditional self-help programs made more internal attributions, 
whereas participants in programs including spouses and medications as part of therapy made more external attributions. Similarly, a study of a traditional inpatient program using the "Minnesota Model" of addiction showed that clients' personal sense of control over their recovery (e.g., internal attributions) increased between intake and discharge (Morojele \& Stephenson, 1992). This program was based upon a disease concept of addiction, although it taught 12-step ideology of responsibility for one's actions. Thus, the expression of internal or external attributions may be a function of treatment teachings as opposed to the occurrence of a lapse or relapse per se. It may be that alcohol and illicit drug users' treatment environments may present more explicit messages that emphasize personal responsibility as compared with those presented in smoking cessation programs.

Thus, the mixed finding among alcoholics and drug users could be a function of the failure to distinguish lapse and relapse or to consider the type of treatment program ideology. In addition. none of the studies reviewed examined the attributions of abstainers following a high risk situation. The present study compared illicit drug users' attributions for abstinence, lapse, and relapse episodes using a precise operational definition of lapse and relapse.

M E T $110 \mathrm{O}$ D $\mathrm{S}$

\section{Participants}

This sample consisted of the first 119 participants, from a larger parent study, the Drug and Alcohol Study (Castro, 1990). The average age of the sample was 29 ( $S D=$ $6.9)$, ranging from 18 to 57 . The sample was primarily male $(61 \%)$. white $(76 \%)$, and earned less than 30,000 dollars per year $(66 \%)$. Admission into the study required that the participant be at least 18 years old and indicate that his/her primary drug of choice was either cocaine $(24 \%)$, methamphetamine $(26 \%)$, or both $(48 \%)$. Persons were not excluded if they reported the concurrent use of other drugs. Of the 111 with complete data regarding other drug use at intake, $83 \%$ had used multiple substances in at least 1 day during the last month. Thus, the majority of the sample were polyusers. Participants with preexisting psychiatric disorders and participants who had been in another drug treatment program within the last 6 months were ineligible to take part in this study.

Participants were recruited from a traditional 28-day inpatient substance abuse treatment center in Southern California. The treatment center utilized typical 12-step ideology, which emphasizes internal responsibility for one's recovery. Treatment included attending individual and group therapy, Alcoholics or Narcotics Anonymous meetings, and didactic sessions. All participants were screened by the treatment center staff before recruitment to the study to assure that they met DSM-IIIR criteria for drug dependence (American Psychiatric Association, 1987).

\section{Procedures}

This paper examines interview data collected 7 months after admission to treatment. Participants were paid 25 dollars for this interview. Eighty-two percent $98 /$ 119) of the original participants were located and interviewed either in person $(n=-$ $86)$ or, due to moving out of state, over the phone $(n=12)$. Twenty-one participants did not complete follow-up interviews and were subsequently dropped from all analyses. 
Attributions. At the 6-month follow-up, attributions were obtained for "first reuse" or "close call but abstinent" situations. Participants were given three questions which were answerable on five-point Likert scales having anchor statements on each end. The locus of causality item stated "Was the cause/reason for your abstinence/ use due to:" $(1)=$ "what you did?" or $(5)=$ "other people or circumstances?'” The stability item stated "Will the cause of the abstinence/reuse be present:" $(1)=$ "in similar situations in the future?" or $(5)=$ "only at that time?" The specificity item stated "Does the cause of the abstinence/use apply:" (1) = "only to this substance?" or $(5)=$ "to other substances?"

Timeline. The Timeline Follow Back protocol was originally developed to examine daily alcohol consumption quantity and frequency, and behavioral aspects of drinking over time (Sobell, Maisto, Sobell, \& Cooper, 1979). In the present study, an identical procedure was used with the addition that the participants provided information about the quantity and frequency of illicit drug use as well as akcohol use. Participants were given a 6-month calendar and were guided to identify the daily quantity and frequency of the use of cocaine, crystal methamphetamine, alcohol, heroin, and marijuana. The timeline calendar protocol has been shown to be a reliable and valid method of assessing drinking behavior using test-retest and convergent methodologies (for a review, see Sobell, Sobell, Leo, \& Caneilla, 1988).

Relapse definitions. Reuse for each of the drugs of interest (cocaine, methamphetamine, alcohol, marijuana, and heroin) and a total for all the drugs, were coded into eight relapse categories from data obtained from the timeline calendar (see Table 2). The eight relapse categories were defined a priori by expert panel view. The distinction between lapse and relapse categories was partially based on the work of Baer, Kamarck, Lichtenstein, and Ransom (1989), who stated that the use of more than one drug during the same week distinguished a "lapse" from a "relapse." Baer's view is congruent with the clinical observation that participants tend to relapse to substances in 2- to 3-day binges. When more than one category was applicable for a given participant, a conservative approach was taken and the more severe category was coded.

Table 2. An eight-level scaling of lapse/relapse outcomes

\begin{tabular}{ll}
\hline Category & \multicolumn{1}{c}{ Definition } \\
\hline Abstinent & Nonuse \\
Lapse-1: Minor & 1 use \\
Lapse-2: Moderate & $2-6$ uses. separated by at least 1 week \\
Lapse-3: Major & $7-26$ uses, separated by at least I week \\
Relapse-1: Minor & $2-3$ uses per week. $\leq 6$ weeks \\
Relapse-2: Moderate & $2-3$ uses per week, $>6$ weeks \\
Relapse-3: Major & $\geq 4$ uses per week, $\leq 6$ weeks \\
Relapse-4: Severe & $\geq 4$ uses per week, $>6$ weeks \\
\hline
\end{tabular}

Note. When a participant fit into more than one category, the more severe category was coded for conservative purposes. 
Psychometric assessment. Reliability of the lapse/relapse categories was determined for a sample of 49 participants. Two independent raters coded the data; percent agreement was .93 . In the event that there was a disagreement between the two raters, a third person blindly rated those cases. If none of the raters agreed, then the choice coded was decided by the first author. While the funding was available, a subsample of the participants' self-report relapse data were validated by a hair testing procedure, radioimmunoassay (RIAH). This procedure has been shown to be reliable in verifying the presence of cocaine, opiate, PCP, and marijuana use for up to 3 months prior to the test date (Baumgartner, Hill, \& Blahd, 1989); this method was concurrently being validated for methamphetamine detection. All participants knew that they may be asked to give a hair sample and that they would be selected at random. Participants did not know whether they would actually be required to give hair until until the interview was completed. If a person did not provide a sample, the next person scheduled for an interview was asked to provide a hair sample.

Overall, of the 74 participants asked to provide a sample, 39 participants supplied hair samples. Of the remaining 35 participants, the hair sample was not obtained for the following reasons: 2 refused to provide hair; 12 were out of town and interviewed by phone; 8 were drop-outs, 4 were not asked due to interviewer error, and 9 had no hair or their hair was too short. Of the 39 participants in which both self-report and RIAH data was available, the concordance rate was $90 \%$ (34/39). Reasons for disagreements were: (a) one participant reported using different drugs than the hair analysis revealed; (b) two participants claimed that they had reused a substance but the hair analysis did not detect any substance use; and (c) one participant claimed sobriety and the hair analysis detected the presence of methamphetamine. Disagreements may be explained by considering quantity, as RIAH is less reliable at detecting small quantities.

\section{Data analysis}

Attributions. A Multivariate Analysis of Variance (MANOVA) was conducted to examine the vector of scores for the three outcome groups (abstainers, lapsers, relapsers) on the attributional dimensions (internal, stable, and global). The assumption was made that the five-point Likert-type attribution scales operated as equal interval measures, that is, as interval-level scales. If the five-point scales were actually ordinal seales. MANOVA is known to be still quite robust despite violating the assumption of internal scaling of the dependent variable (Bray \& Maxwell, 1985).

\section{R E, S U I, T S}

Demographic and rease. The number of days of substance use within the previous 6 months was not significantly associated with any demographic variables, including age, gender, income. or ethnicity. When correlating days of reuse and lifetime use of alcohol, heroin, cocaine, amphetamines, and marijuana. only the greater number of years of lifetime heroin use was correlated with greater number of days of substance use during the follow-up $(r=.26, p<.05)$; however, only a small proportion of the participants used heroin. The greater the number of times treated for drugs or alcohol was related to a greater number of days of substance use during the follow-up $(r=.32, p<.01, r=.38, p<.01$, respectively $)$. 
Table 3. Percentage of abstinent, lapsed, relapsed participants by specific substances

\begin{tabular}{lcccccr}
\hline Category & Any & Coc & Met & Alc & Mar & Her \\
\hline Abstinent & 42 & 80 & 79 & 55 & 73 & 97 \\
Lapse & 29 & 13 & 12 & 28 & 16 & 1 \\
Relapse & 30 & 7 & 9 & 18 & 9 & 2 \\
\hline
\end{tabular}

Note. See Table 2 for explanation of lapse and relapse levels.

Any $=$ any substance, $\mathrm{Coc}=$ cocaine, $\mathrm{Met}=$ methamphetamine. Alc $=$ alcohol, Mar = marijuana, Her $=$ heroin.

Abstinence, lapse, and relapse. For the 98 participants that were located at followup, $42 \%$ were abstinent for the entire 6 months, $29 \%$ lapsed, and $30 \%$ relapsed. Table 3 shows the percentage of participants who were abstinent, lapsed, or relapsed to a particular substance (cocaine, methamphetamine, marijuana, alcohol, or heroin). Notice that the percentage of participants who lapsed to a particular drug is greater than those who relapsed to a particular drug (either cocaine, alcohol, methamphetamine, or marijuana). The overall percentage of participants who lapsed to any substance, however, is similar to the number who relapsed. Together, these two observations suggests that those who relapse are more likely to use multiple substances as compared to those who lapse. Table 4 shows the more detailed eight lapse-relapse categories for "any drug" and for each specific substance. Notice that the distributions are highly skewed given the number of participants who remained abstinent. Further, very few participants were categorized as "moderate relapsers" (2-3 days per week for more than 6 weeks). If relapsing for a period of more than 6 weeks, the number of days of use increased from 2 to 3 days a week (minor relapse) to at least 4 days a week (severe relapse). Similarly, if relapsing for the period of less than 6 weeks, the number of days of use per week was more often 2 to 3 days of use (minor relapse), rather than 4 to 5 days a week (major relapse).

Attributions. Three attributional dimensions were examined as dependent measures using a MANOVA with relapse status (abstinence, lapse, relapse) serving as the independent variable for the 97 participants for which complete data was avail-

Table 4. Percentage of participants' specific substance use according to the detailed 8-level lapse/relapse categories

\begin{tabular}{lrrrrrr}
\hline Category & Any & Coc & Met & Alc & Mar & Her \\
\hline Abstinent & 42 & 80 & 79 & 54 & 75 & 97 \\
Lapse 1: Minor & 9 & 3 & 3 & 8 & 4 & 0 \\
Lapse 2: Moderatc & 11 & 8 & 6 & 11 & 9 & 1 \\
Lapse 3: Major & 8 & 2 & 3 & 9 & 3 & 0 \\
Relapse 1: Minor & 10 & 3 & 4 & 4 & 2 & 1 \\
Relapse 2: Moderate & 0 & 0 & 1 & 1 & 1 & 0 \\
Relapse 3: Major & 4 & 1 & 2 & 3 & 1 & 0 \\
Relapse 4: Severe & 15 & 3 & 2 & 9 & 5 & 1 \\
\hline
\end{tabular}

Note. See Table 2 for explanation of lapse and relapse levels.

Any $=$ any substance, $\mathrm{Coc}=$ cocaine, Met $=$ methamphetamine, Alc $=$ alcohol, Mar = marijuana, $\mathrm{Her}=$ heroin. 
Table 5. MANOVA: Relapse outcome by attributions $(n=97)$

\begin{tabular}{|c|c|c|c|c|c|c|c|}
\hline \multirow[b]{2}{*}{ Variable } & \multicolumn{3}{|c|}{ Group } & \multirow[b]{2}{*}{$F$-1est } & \multicolumn{3}{|c|}{ Group contrasts } \\
\hline & $A$ & I. & $\mathrm{R}$ & & A vi. I. & L w R R & A $v \mathrm{~s}, \mathrm{R}$ \\
\hline 1 & 4.12 & 3.25 & 3.57 & $3.49 \%$ & 2.57 & -0.87 & 1.62 \\
\hline$S$ & 4.05 & 2.75 & 3.71 & 5.72 & $3.34 \%$ & -2.27 & 0.86 \\
\hline G & 4.20 & 1.86 & 3.11 & $19.12^{2}$ & $6.17 \ldots$ & 3.03 & $2 \times 7: \%$ \\
\hline
\end{tabular}

Note. Groups are: $\mathrm{A}=$ Abstinent. $\mathrm{L}=$ Lapse. $\mathrm{R}=$ Relapse. Scale score ranges for 1 : $(1)$ external. $(5)$ internal: for $S:(1)$ unstable. (5) stable: for $(\mathrm{j}:(1)$ specific, (5) global.

$* p<.05$.

$* * 0.01$.

* : $p<.001$

able (see Table 5). The overall Wilks' lambda for the effect of relapse group was significant (Wilks' lambda $=.68$, approximate $F=6.52, p<.001$ ). When examining the Roy-Bergman step-down $F$ tests, the "global" attributional dimension was the strongest contributor to overall group differences $(F=19.12, p<.001)$. The "stable" $(F=5.72, p<.01)$ and "internal" $(F=3.49, p<.05)$ attributional dimensions were also important contributors to the main effect. Planned group comparisons were next conducted to compare the attributions of abstainers, lapsers, and relapsers in order to evaluate the proposed pattern of relationships that are shown in Table 1.

Overall, the results offer corroboration for the patterns of attributions postulated for abstainers as presented in Table 1, and offer partial corroboration for the patterns postulated for lapsers and relapsers.

Regarding the dimension locus of causality, there was one significant group contrast; the abstinent group $(M-4.12)$ exhibited higher internal scores as compared with the lapse group $(M=3.25)$. Thus, the abstainers strongly attributed their success in avoiding relapse to themselves. By contrast, the lapsers were more ambivalent regarding the cause of their use as indicated by their attributions score. which was near the midpoint 3.00 but still on the internal side of the scale. The relapsers viewed their relapse (failure to maintain abstinence) as internally caused - which was not significantly different than the abstainers or the lapsers. In summary, all groups made attributions that were more internal than external in perceived locus of causality (all means were greater than 3.0).

Regarding the dimension of stability, the abstainers $(M=4.05)$ made more stable attributions regarding their success, compared with the lapsers $(M=2.75)$ who had a slight tendency to make unstable attributions for their use. Also, the relapse group $(M=3.71)$ made stronger stable attributions as compared with the lapse group $(M=$ 2.75). Similar to the abstainers, the relapsers perceived their relapse as a trait-like disposition, one that remains stable across time. Thus, the abstainers $(M=4.05)$ and the relapsers ( $M=3.71$ ) made equally strong stable attributions, although for completely different reasons.

Regarding the dimension of specificity, the abstainers $(M=4.20)$ made global attributions, as compared with the lapsers $(M=1.86)$ who made specific attributions. The abstainers perceived that their abstinence was likely to occur regardless of substance, while the lapsers perceived that their slip was specific to a given substance. The relapsers $(M=3.11)$ expressed significantly less specificity in their attributions for reuse than the lapsers and significantly more specificity than abstain- 
ers. Thus, with scores near the scale's midpoint (3.00), relapsers were unsure whether future relapses would occur to the same substance or a different substance. This suggests that relapsers viewed their relapse behavior with uncertainty as related to the use of other drugs, while lapsers viewed their brief use as an event that resulted from cues that were specific to the drug which they used.

DISCUSSION

\section{Key findings}

One contribution from the present study is added precision in the categorization of lapse and relapse, which provides the tools for more precise comparisons of the attributions for various levels of relapse behavior and facilitates comparisons of results from one study to another. The detailed manner in which reuse was defined has provided new information about the pattern in which lapses and relapses occur. A key finding from this study is that the theorized pattern of attributions among abstainers, as expanded from Marlatt and Gordons' (1985) relapse model, was empirically validated among substance users. Support for the validity of the Abstinence Violation Effect as a determinant of whether a person relapses or abstains, once lapsing, was mixed. Lapsers and relapsers were primarily distinguished by the specificity and stability of their attributions.

\section{Relapse characteristics}

The substance most frequently used following treatment in this sample was alcohol. This may be explained by the social acceptability and accessibility of alcohol as opposed to illegal drugs. Does this imply that participants replace their drug of choice (cocaine or methamphetamine) with alcohol, or that alcohol is a "gateway" drug for relapse? Some participants were using alcohol infrequently; 27\% lapsed to alcohol (once a week or less of use). Anecdotally. many participants claimed that they could use alcohol without problems. This is in contrast to research findings that have shown a correlation between alcohol use and relapse among cigarette smokers (Brandon, Tiffany, Obremski, \& Baker, 1990). While over $80 \%$ of the participants used multiple substances before treatment, only $12 \%$ used more than one drug at follow-up (either cocaine, crystal, and/or marijuana) and $17 \%$ used alcohol and one drug at follow-up (either cocaine or crystal). Additional longitudinal research is needed regarding the natural history of relapse in order to determine what proportion of the lapsers to alcohol (as well as those who lapsed to other substances) eventually relapse to alcohol or to their original drug of choice.

\section{Attributions}

The role of attributions, suggested by the Abstinence Violation Effect, in determining reuse outcome was partially confirmed. Results for the stable dimension directly confirmed the hypotheses outlined in Table 1. Abstainers and relapsers made stable attributions as predicted, albeit for different reasons. Abstainers believed that their ability to abstain would continue in similar situations in the future: relapsers believed their reuse would occur in similar situations in the future. In contrast to relapsers and abstainers. lapsers indicated that their slip was less likely to occur in similar situations in the future. Examination of lapsers ${ }^{\circ}$ average response, which was near the middle of the stable/unstable dimension. showed that their response is 
actually an ambivalent one. The finding that lapsers and relapsers make different attributions regarding whether their substance use would continue is consistent with prior findings among smokers (Curry et al., 1987).

The hypotheses in Table 1 for the specificity dimension were confirmed for $a b$ stainers and lapsers. To illustrate, abstainers thought that their success at abstinence would persist regardless of the substance available (a global attribution). Results were also confirmed for lapsers as they indicated that their slip was essentially a "unique" event such that their brief use was not likely to transfer to the use of other substances. The hypothesis that relapsers, as compared with lapsers. would make stronger global attributions (that their use would transfer to use other substances) was also confirmed. These findings are also in accordance with the results of Curry et al. (1987). who reported that cigaretle smokers who relapsed made more internal, stable, and global attributions than did lapsers.

Unexpectedly, on the specific-global dimension, relapsers' scores were significantly different from those of the abstainers. It was expected that relapsers would view their relapse globally. that is. that their use would occur regardless of type of drug, since they would sec their use as a trait-like characteristics. Upon examination of the average response, relapsers are ambivalent as to whether their use is specific to one substance or would generalize to multiple substances. Perhaps this finding reflects the severity of the relapse episode. As substance abusers' relapses becomes more severe, they may be more likely to begin using multiple substances based on substance availability or degree of dependence. This explanation is partially supported by the finding that the attributional processes of smokers vary according to degree of physical dependence (Martin, 1990).

The findings for the "locus of causality" dimension only partially confirmed the predictions offered by the AVE. but in a different pattern than for specificity. As expected, abstainers and relapsers accepted individual responsibility (internal attributions) for their abstinence or reuse in a tempting situation. Lapsers, however, were predicted to distance themselves from their slip by attributing it to external causes according to the AVE. Results showed that lapsers made significantly different attributions regarding locus of causality than did abstainers. although the attributions of lapsers did not differ from those of relapsers. Examination of the mean score. however, shows that lapsers were closer to the internal pole of the scale than to the external pole. Thus, all three groups made internal attributions, although the degree varied. This is in contrast to the findings among cigarette smokers reported by Curry et al. (1987) and O'Connell and Martin (1987).

The clustering of scores in the internal range for the locus of causality attribution could reflect the treatment ideology that was offered by the inpatient 12-step program from which the participants were recruited. After all, studies have shown that treatment programs can influence the attributions of illicit drug users for abstinence or reuse (Harackiewicz et al., 1987: Maisto et al., 1988: Morojele \& Stephenson. 1992). The program from which the participants of the present study were recruited advocated that clients take personal responsibility for their recovery (Castro, Sharp. Barrington, Walton, \& Rawson, 1991).

\section{Limitations}

Despite offering several important findings, there are limitations to this study. The sample is mainly composed of White males from middle class backgrounds. While this demographic profile may be reflective of many inpatient substance abuse treat- 
ment populations, these results should not be generalized to other populations (e.g., women, minorities). This caution is especially warranted since studies have found that attributional processes vary in relation to ethnicity (Crowder, 1982) and gender (Gutierres \& Reich, 1988). This study is, however, one of the few attribution studies conducted among illicit drug users. While self-report was used in this study, every attempt was made to obtain valid results including: (a) using reliable measures. (b) emphasizing confidentiality, and (c) informing participants prior to each interview that some of the self-reports would be validated via hair analysis. Finally, no attempt was made to control for the associations between heroin use and relapse and number of previous treatment episodes and relapse. The reason for the former is that very few participants used heroin pre-treatment or relapsed to use heroin. The reasons for the latter are that any participation in previous treatment programs occurred at least 6 months prior to this treatment episode. and that no information was available as to the type of the program or its treatment ideology. Future studies might obtain a more detailed treatment history to tease out these potential sources of confound.

\section{CONCLUSIONS}

Is the Abstinence Violation Effect empirically valid among illicit substance users? These results provide partial support for the predictions made by the Abstinence Violation Effect. Success in avoiding temptation to return to the use of substance (complete abstinence) was associated with making internal. stable, and specific attributions. Thus, successful participants perceived their abstinence as being "caused" or effected by skills or abilities to resist temptation. and that these capabilities are influenced across situations and across time. It appears that abstinence affords the recovering substance user a sense of empowerment over substances.

Relapsers perceived their failure to abstain as internally caused, as likely to occur again in various situations in the future, and as possibly transferable to multiple substances. Clearly, this is a most negative psychological outlook for the recovering substance user. Perhaps this attributional pattern reflects a belief among relapsers in outcome as a trait that they cannot control regardless of situation or effort. In contrast, lapsers attributed their use more to situational factors that would not be present in the future and that were restricted to a specific drug. Thus, lapsers could maintain the view that their minor transgression was a limited one, and not a broadbased transgression as was apparent among relapsers.

Any inconsistencies found among lapsers may also relate to other factors that serve as determinants of attributions. such as the doctrine emphasized by the treatment program. Additionally, it could be that the laser group may consist of two subgroups of people: (a) those who will recover and remain abstinent after their limited slip (by attributing their lapse to lack of effort), and (b) those who will falter and eventually relapse (by attributing their lapse to a personal trait). If this post-hoc explanation is true, examining the attributions of lapsers could assist clinicians in identifying clients who were headed for a full-blown relapse (i.e., the latter group), so that preventive interventions could be implemented.

Future studies might also determine whether the lapsing persons consider their lapse a success or a failure. The assumption is made in the literature that participants view abstinence as a success whereas a lapse is viewed as a failure (to remain sober). This conception is reflected by many treatment programs whose goals are complete abstinence from alcohol and drugs. Yet. some participants may not view their lapse 
as a failure. Several participants, whose primary drug of choice was a stimulant, remarked (to the first and third authors) during interviews that their lapse to alcohol was nonproblematic for them. They indicated that they always intended to use alcohol since they did not believe that they were addicted to it. Their attributions for their lapse to alcohol (which they may not regard as a failure at maintaining abstinence) are likely to be very different from the attributions of the person who views himself as addicted to alcohol, such that any return to alcohol use is interpreted as at failure. Thus, future studies might better explain the role of the attributional process in treatment outcome by asking the participants not only about their attributions for their behavior (i.e., abstinence, lapse, or relapse), but also about their abstinence goals. This will help determine whether the participant considers the use of a particular substance (c.g., alcohol) as problematic, and, whether or not they consider a lapse to that substance a failure.

\section{M P L I C A I O N S}

Since studies show that treatment programs can influence the attributions of their participants, what attributions would be best for relapse-prevention programs to emphasize? Treatment programs may be most effective if they include reattribution training (Fiske \& Taylor, 1991) as a key component of relapse prevention. Among substance abusers, a self-serving attributional bias (Fiske \& Taylor, 1991) may be adaptive as abstainers could be taught to make different attributions (internal, stable, and global) than lapsers (external, unstable, and specific) in order to prevent relapse. Further understanding of the role of attributions in the relapse process is vital in order to increase the effectiveness of substance abuse treatment and to reduce the health care costs of recidivism.

R E F E R E N C F S

Abramson. L. Y., Garber, J., \& Seligman, M. E. P. (1980). Learned helplessness in humans: An attributional analysis. In J. Garber \& M. E. P. Sleigman (Eds.). Human helplessness: Theory and application. (pp. 3-34). New York: Academic Press.

American Psychiatric Association. (1987). Diagnostic and statistical manaal of memal disorders (Brd ed.). Washington. DC: Author.

Baer. J. S.. Kamarck, R., Lichtenstein. E.. \& Ransom, C. C. (1989). Prediction of smoking relapse: Analysis of temptations and transgressions after initial cessation. Jommal of Comsalting and Clinical Prychology, 57(5), 623-627.

Baumgartner, W. A., Hill. V. A.. \& Blahd, W. H. (1989). Hair analysis for drugs of abuse. Journal of Forensic Sciences, 34(6), 1433-1453.

Birke. S. A., Fdelmann. R. J.. \& Davis. P. E. (1990). An analysis of the abstinence violation effect in a sample of illicit drug users. British Journal of Addiction, 85. 1299-1307.

Bradley, B. P., Gossop. M., Brewin, C. R.. Phillips, G., \& Green. L. (1992). Attributions and relapse in opiate addicts. Journal of Consulting and Clinical Psychology, 60(3), 470-472.

Brandon, R. H., Tiffany. S. T.. Obremski, K. M. \& Baker, T. B. (1990). Postcessation cigarette use: The process of relapse. Addictive Behaviors, 15. 105-114.

Bray, J. H., \& Maxwell. S, E. (1985). Mulhivariate analysis of variance. Newbury Park, CA: Sage.

Brownell, K. D., Marlatt, G. A.. Lichtenstein. E., \& Wilson. G. T. (1986). Understanding and preventing relapse. American Psychologist, 41(7). 765-782.

Castro. F. G. (1990). Drig and alcohol study profocol. Unpublished manuscript.

Castro. F. G.. Sharp, E. V., Barrington. E. H., Walton, M.. \& Rawson. R. A. (1991). Drug abuse identity in Mexican Americans: Theoretical and empirical considerations. Hispanic Jounal of Behavioral Science, 13, 209-225.

Crowder, R. L. (1982). Methadone-maintained males' reactions to success and failure: Causal attributions, self-efficacy, and attitudes toward heroin. Drag and Alcohol Dependenee, 10, 367-381.

Curry. S., Marlatt, G. A., \& Gordon, J. R. (1987). Abstinence Violation Effect: Validation of an attributional construct with smoking cessation. Journal of Consulting and Clinical Psychology. 55(2). 145149. 
Fiske, S. T., \& Taylor, S. E. (1991). Social cognition (2nd ed.). New York: McGraw-Hill.

Goldstein, S., Gordon, J. R., \& Marlatt. G. A. (1984, August). Attributional processes and relapse following smoking cessation. Paper presented at the American Psychological Association Convention, Toronto. Ontario, Canada.

Gutierres, S. E., \& Reich. J. W. (1988). Attributional analysis of drug abuse and gender: Effects of treatment and relationship to rehabilitation. Journal of Social and Clinical Psychology. 7, 176-191.

Harackiewicz. J. M., Sansome. C.. Blair. L. W., Epstein. J. A.. \& Manderlink. G. (1987). Attributional processes in behavior change and maintenance: Smoking cessation and continued abstinence. Journal of Consulting and Clinical Psychology. 55(3), 372-378.

Hubbard, R. L., \& Marsden. M. E. (1986). Relapse to use of heroin, cocaine, and other drugs in the first year after treatment. National Institute on Ditug Abuse Research Monograph Serics. 72 . Rockville. MD.

Hunt, W. A.. Barnett, L. W., \& Branch. L. G. (1971). Relapse rates in addiction programs. Jomrnal of Clinical Psychology, 27, 455-456.

Maisto. S. A.. O'Farrell. T. J.. Connors. G. J.. Mckay. J. R., \& Pelcovits. M. (1988). Alcoholics' attributions of factors affecting their relapse to drinking and reasons for terminating relapse episodes. Addictive Behaviors, 13(1). 79-82.

Marlatt, G. A., \& Gordon, J. R. (Eds.). (1985). Relapse prevention: Maintenance strategiess in addictive behavor change. New York: Guilford.

Martin, D. S. (1990). Physical dependence and attributions of addiction among cigarette smokers. Addictive Behaviors, 15, 69-72.

McKay, J. R., O'Farrell, T. J., Maisto, S. A.. Connors, G. J., \& Funder, D. C. (1989). Biases in relapse attributions made by alcoholics and their wives. Addictive Behaviors, 14. 513-522.

Morojele. N. K.. \& Stephenson. G. M. (1992). The Minnesota Model in the treatment of addictions: A social psychological assessment of changes in beliefs and attributions. Journal of Community and applied Social Psychology, 2, 25-41.

O'Connell, K. A. \& Martin. E. J. (1987). Highly tempting situations associated with abstinence, temporary, lapse, and relapse among participants in smoking cessation programs. Journal of Consulting and Clinical Psychologs. 55(3). 367-371.

Sobeli. L. C., Maisto. S. A., Sobell. M. B.. \& Cooper. A. M. (1979). Reliability of alcohol abusers" selfreports of drinking behavior. Behavior Research and Therapy. 17. 157-160.

Sobell. L. C.. Sobell, M. B.. Leo. G. I. \& Caneilla. A. (1988). Reliability of a timeline method: Assessing normal drinkers reports of recent drinking and a comparative evaluation across several populations. British Journal of Addiction, 83, 393-402.

Wallace, B. C. (1989). Psychological and environmental determinants of relapse in crack cocaine smokers. Jolurnal of Substance Abuse Treatment, 6, 95-106.

Washton. A. M. (1986). Nonpharmacologic treatment of cocaine abuse. Psychiatric Clinics of Nonth America. 9(3), 563-571.

Weiner. B. (1974). Achievement motivation and atribation theory. Morristown. NJ: General Learning Press. 